\title{
EVOLUTIONS IN INTER-YEAR TEAM WORKING: A LONG-TERM CROSS-SECTIONAL VIEW
}

\author{
Clare GREEN \\ ISD Rubika, Valenciennes, France
}

\begin{abstract}
Team-working remains a core feature of design-related activity. As design methodology expands beyond traditional practice contexts, designers' ability to work in complex multi-disciplinary teams and collaborative environments is increasingly relevant. This paper re-evaluates a series of inter-year team project modules introducing students to collaborative working. Carried out from 2010 to 2013, previous research explored how past teamwork experiences influence future team behaviour and what learning is reapplied. This led to identification of difficulties and moderating factors in these projects, and subsequent improvements in the modules and advice given to students. New research carried out from 2019 to 2020 confirms previous insights into positive reported impacts on personal student evolution and better understanding of good teamwork practice. At the same time the experiential learning identified by students has changed, and issues such as time-related stress, divergence of experiences and passion/motivation are emerging. While these findings relate to team-working in one design school, the prolonged experience of heterogeneous team-working in the school and the cross-sectional approach help to highlight emerging issues that may be important design education in the future.
\end{abstract}

\section{Keywords: Inter-year teamwork, heterogeneous teams, experiential learning, reflective learning}

\section{INTRODUCTION}

Team-working remains a core feature in much of design activity, and although the higher education context may not fully replicate the diversity encountered in the design workplace, it can give students concrete experience preparing them for heterogeneous team-working [1]. This paper re-evaluates a series of inter-year team project modules introducing students to advantages and difficulties involved in collaborative working. Research based on retrospective student feedback carried out in 2012/13 highlighted strengths and weaknesses of this module as a learning experience. The same protocol was used in new research (2019/20) enabling comparisons and identification of evolutions.

'Design Thinking' has become an over-used term, but the key aspects described in literature [2] are very similar to the overall goals and advantages of the consecutive team-working projects. Points noted by Rauth \& Jobst, [2] closely match learning through successive team-working practices:

- Human-centred (emphasis on people as sources of inspiration for solving design challenges),

- Mindful of Process (becoming progressively aware of processes through repeat projects),

- Empathy (identification with/experiencing people's feelings, team-members/user focus of projects),

- Show Don't Tell (recognising importance of diverse communication [3] in effective team-working)

- Radical Collaboration (diverse multidisciplinary teams, examining and reflecting on team dynamics). Authentic teamwork competencies help equip students for increasingly complex working environments [1] and team-working skills are widely appreciated by external school partners and employers of the school's graduates. This research gives insights on issues that may affect the continued quality of these modules as experiential learning, in a rapidly evolving educational context.

\section{SPECIFIC CONTEXT \& RESEARCH METHODS}

The specific repeated teamwork module studied here is a key element of design studies from years three to five in master's courses in Product, Service and Transportation design. Each team project involves two or more-year groups, as well as students from between two and four different specialisations and lasts for 4 to 5 months. Projects involve teams of third- and fifth-year students working together (3|5 teams) in the first semester, followed by third- and fourth-year student teams in the second semester (3|4 teams). Each student thus experiences four inter-year team projects over their final three years of study 
$(3|5,3| 4,4 \mid 3$ and 5|3). Two previous studies in 2012 and $2013[4,5]$ highlighted that this cumulative teamwork experience appears to have created strong team-working/team-leading skills in final year students by allowing students to gradually build up team-working knowledge [3].

Teamwork processes in further education can be described as essentially encompassing reaching consensus on how decision making will occur; interpersonal communication; collaborative problem solving; and conflict resolution [1]. While these four points give a sound, start point for addressing teamworking, to study how effectively team-work processes are being learned, a model addressing the complexity of team-working can be useful. Tucker et al. [3,6] identify 22 different factors in successful team-working, in a similar educational context (architecture/art and design schools in Australia). Team sizes (average five members) advocated in this research match those occurring in the school.

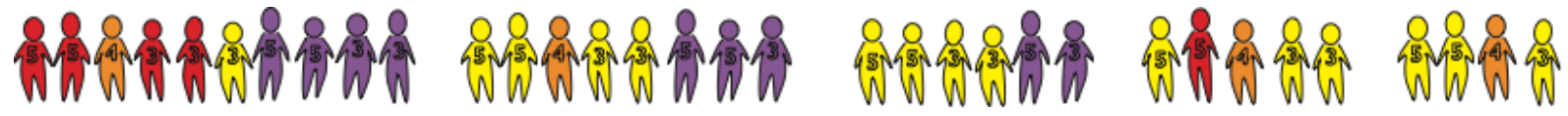

Figure 1. Examples of five actual teams in the 2019-2020 cohort, 3/5 projects (red indicates transport students, orange: students, yellow: product students, violet: digital modellers)

Figure 1 gives examples of team make-up encountered by students and shows their heterogeneity. Students do not have specific theoretical teaching in teamwork process but do have tutor support during the projects related to both project outcome and team-working issues. While students do not follow a formal team-building process [7], the first day of their projects is assigned to team-building activities. Teams are encouraged to do ice-breaking activities, establish goals/purpose, exchange reflections on past projects, identify individual wishes/roles and set up their workspaces. These points mostly match actions suggested by Kayes \& Kolb [7], (their "workbook" takes a similar timing).

While the overall teamwork modules and cumulative learning experience appear to continue to be effective, the current study aimed to investigate if new issues are emerging in student experiences of these projects, in the light of increased diversity in the student cohort, and in evolutions that may be present in student profiles and concerns.

The research methods to study evolutions in the student experience of these inter-year projects are both cross-sectional and longitudinal [8]. Retrospective reports, written by students in their final year describe their experiences during their "journey" through four consecutive long projects. The same protocol and questions were used for school years 2012/2013 and 2019/2020. In 2012/2013, 43 out of 57 students (the entire year group) returned detailed replies. In the 2019/2020 cohort 19 out of the total 35 students replied. In addition, retrospective reports by third year students working on the same teams as the fifth-year students allow the viewpoints and experiences of junior team members in these collaborative team-work projects to be considered. Third year retrospective reports were written in the context of, and preceding, an in-class discussion of team-working practices. Writing on their first long project experience, the 3rd year cohort included 44 students (34 gave written feedback).

\section{LEARNING EXPERIENCES IDENTIFIED BY STUDENTS}

The experiential learning, in the sense of knowledge created through the transformation of experiences [7] identified by students through their own team-work journeys is very diverse. The scope of this article does not permit covering every theme in detail. It is nevertheless possible to group the themes into two main families: those relating to personal evolution/skills acquired not directly related to team-working practices, and those linked to team-working skills. It is also important to note that there is a difference between what students' label "learning" in their self-reports, and what learning appears through analysis of more general comments and anecdotes in the retrospective reports.

\subsection{Personal evolution}

A third year student notes after finishing his $3 \mid 5$ long project: "I came out of this project having grown, with people who at the start were strangers and who have become close friends...I learnt enormously". The report suggests teamwork involving getting to know team members personally, but the "learning" commented on by the student is more in terms of processes and skills. This quote from the 3rd year cohort, matches the place of learning "craft" skills and methodology in 5th year reports. Most comments relating to learning skills relate to the first long project (3|5). Learning methodology is one of the most recurring themes: "I learned good design methodology, teamwork and to go into the details. I developed my critical point of view, my design sensitivity and my design process." Or: "I learned enormously on 
the way to carry out a project, to construct a presentation." Beyond method and process, many comments relate to learning specific skills and tools, such as software, sketching, mood boards. Craft skills were more mentioned by the 2012/13 cohort, and methodology by the 2019/20 students. A third theme, present in both studies is personal evolution in attitude, with comments on patience, being more open to criticism, self-reliance, autonomy, self-confidence...

\subsection{Teamwork understanding}

The experiential learning identified by 5 th year students can be compared to best practice in team working [3,6] and conflict management [9]. Some essential team-working skills identified [3] are coordination of tasks, (diversified) communication, effective team-based ideation and idea selection, decisionmaking, leadership and conflict management. In turn, conflict management [9] relates back, in part; to time management, forecasting; understanding members' skills and individual interests and generally to understanding/awareness of diversity which can improve empathy, and communication. Equally important for pre-empting conflict is taking a "whole-group" perspective in establishing processes that apply to everyone and are for the benefit of the task performance [9].

Student reports illustrate examples of many good team-work practices with themes related to communication and effective time-management/organisation the most directly identified as learning. A transport student mentions that in team working it is "Nice, cool to share points of view" and adds that it is important to talk to "avoid tensions accumulating through the project". Another student mentions she learned that "disagreeing and having different opinions is not a weakness".

Many students mention learning the importance of group cohesion, often linked to how a friendly, enjoyable atmosphere facilitates group working. One comment links organisation and good atmosphere: "I've learnt to be organised in my work, respect the schedule according the deadline we had. Work in a really fun mood while focusing on our work". Another student links several themes: "I understood how important it is to make sure everyone is involved in the team, and how positively it can impact our work" Some students mention learning good process for team-based ideation: "I've learnt a lot about this point especially during the creativity phase, where we discussed a lot regarding ideas, try to push more our concept thanks to the idea of someone else." Another student learned to "value each of the ideas proposed by my comrades in order to let the reflections grow". Comments show this theme to also be a source of conflict, and being present in few reports, this is an area for more tutorial/teaching support and improvement.

Already mentioned in previous research [5], and reconfirmed here, is a "learning frame of mind". Students comment learning from their peers and from junior team members. These comments come from students whose projects $(\mathbf{5} \mid 3)$ demonstrated genuine team-working/team-leading skills. This theme is not directly identified by students as learning but appears in analysis of their reports.

\section{EMERGING TEAMWORKING ISSUES}

The following themes emerge as evolutions in relation to experiences reported in the earlier research.

\subsection{Heterogeneity}

Individual differences in teams have been identified as a key issue contributing to negative teamwork experiences [6]. Heterogeneity is growing in the design school cohort, with an increasingly international student intake and new specialisations being introduced. A 3rd year female student noted one of her team leaders did not address a male junior team member in the same way as her "he said to [male student]... "Could you please do that" and said to me "You do that". It was a bit worrying for me working with him because you could feel that he didn't consider me as equal to [male student]" Cultural diversity in the student cohort generates new inter-personal issues that the school may not have been fully aware of or prepared for.

Heterogeneity in terms of skill level is inherent in the team-working format at the centre of this research. Some 5th year students comment difficult experiences working with perfectionist final year students while in their 3rd year. Mainly present in $\mathbf{3} \mid 5$ teams, students keep negative impressions of these projects despite identifying having learned valuable skills and methodology. One student writes: "It made me very exigent toward the result. It is good because it helps me to improve myself and my work, but sometimes it stresses me too much. And because I had a bad experience with a strict and hard management, I am always afraid that the third years in my group might feel bad". 


\subsection{Hierarchies}

While the four-project model creates a progressive learning context, it also generates expectations around personal evolution from junior team member to senior team member, and more problematically from "member" to "manager". Previous findings [4,5] indicated non-productive hierarchies generating team problems. Despite changes in team-working advice given to students, this remains an issue for some students in their team-leading roles. New findings highlight both problematic hierarchies on one hand and successful non-hierarchical teams on the other, suggesting 1) emphasis should be "team-working", 2) senior members should aim for (team) leadership rather than (team) management.

Clearly some 5th year students have developed team-leading skills avoiding problematic hierarchies, illustrated by the following quotes by 3rd years in 2019/2020: "we wanted to work with him (and not for him) because he helped us to like our project and helped us to always share our opinions". Another comments "everyone knew what he had to do...the 5th years didn't impose their ideas on us, quite the opposite. We discussed all the time to find the best solutions (which all the group liked). No leader or manager, each of us were." Opposed to this, in another group, the junior student comments "decisions were taken unilaterally...I don't have the impression that the final project is the result of my work" and another student mentioned "Our role for this phase ended up consisting in trying to find justifications for the choices that were taken behind the screen of our manager". Managers here had not created "positive interdependence and a collective pursuit of goals and objectives" [6], which could positively contribute to group cohesion.

\subsection{Diverging experiences}

Student experiences illustrate different forms of divergence. All reports on student journeys express diverging experiences of team-working in the long projects. All students report at least one globally positive experience out of their four projects, and over half mention one negative experience.

The second form of divergence relates to differing appreciations of the same long-project team. The most marked example of this point relates to differences between 3rd and 5th year experiences of the same projects. Taken overall, 3rd year experiences of their first long project for the 2019/2020 cohort were systematically the same or less positive than those of the 5 th years in the same groups.

Table 1. reported experience from 5th and 3rd year student viewpoints

\begin{tabular}{|c|c|c|c|c|}
\hline experience of 315 proiect & 5th vear $(n 19)$ & 5 th vear as \% & 3rd year $(n 34)$ & 3rd vear as \% \\
\hline excellent & 1 & 5 & 1 & 3 \\
\hline positive & 11 & 58 & 11 & 32 \\
\hline mixed & 6 & 32 & 14 & 41 \\
\hline negative & 1 & 5 & 8 & 24 \\
\hline
\end{tabular}

Two points mediate this result. 1) Successful team leaders (positive personal experiences, positive feedback from 3rd year team members and good grades for the project) had themselves one negative team working experience (one in each case for the three journeys identified). While the sample size does not allow for robust conclusions, findings also illustrate that two of the "managers" with whom junior members signalled unsatisfactory team-working experiences, had not themselves experienced a negative team project. 2) Reported experiences of 5th year students on their own junior member experiences are very similar to current 3 rd year student experiences. Their own $3 \mid 5$ experiences can be classed as $41 \%$ positive, $35 \%$ mixed and $24 \%$ negative, and their $3 \mid 4$ experiences as $47 \%$ positive, $29 \%$ mixed and $24 \%$ negative. None of the 5th year students reported two successive negative project experiences. Negative experiences may be less well accepted by the recent cohort (based on 3rd year replies). Reflection, when possible, can to a certain extent help retrospectively give value to less positive experiences [6]

Beyond overall project evaluations, individual appreciations on specific moments of team-working process can be very different. A 5th year student mentioned her planning "with detailed tasks... to know who has to do what. This system works very well because it allows us to be satisfied when a task is "crossed out" but also allows the third years to follow the progress easily". A junior member of the same team retrospectively commented almost the opposite "the lack of retro-planning to know all the work to be done didn't help us to keep up a good work rhythm because we never knew in advance what we needed to do." Reports by two 5 th years on the same $3 \mid 4$ project are also revealing, one mentioning 
"everybody was really motivated from the beginning to the end", while a fellow team-leader writes "to work with people who don't share the same passion as you is really complicated".

\subsection{Passion, motivation and enjoyment}

As active learning, team-based projects are generally associated with increased motivation and engagement from students [10], but lack of motivation was an issue identified in the 2013 study. In the 2019 study, fellow student lack of motivation is less mentioned, but motivation as a desirable group dynamic is commented on (by over half of 5th years). For example "We had a hard time moving forward at times, but everyone remained motivated and we learned a lot," in a $4 \mid 3$ project or "It was really easy to work with this team, everybody was really motivated by the project" in a $\mathbf{5} \mid 3$ experience. Motivation indicates group effectiveness for students and is something they are actively concerned about. References to students abandoning teams is a related issue does not present in the earlier cohort

The notion of "passion" is much more present in the recent study, both from 3rd and 5th year students (19\% of 5th year students mention passion in 2012/2013, 31\% in 2019/2020). For example "it was a pleasure for me to come to school every day to share my passion..." (5|3 experience) or on the long projects as a whole: "The positive elements that I would draw from these projects are first of all meeting people who share the same passion as me." Both year groups' comments use the term passion for a deep individual engagement which indicate pleasure or its lack in team working. "These long projects push us to produce strong, complete and developed solutions but when the passion isn't there it's a heavy weight to bear" (comment by a junior member in 2019/2020). Along the same lines, references to fun, enjoyment and pleasure are considerably more present in the 2019/20 5th year cohort (68\% of students compared to $43 \%$ in 2012/2013). The language used for personal experiences has become more emotional, which may reflect an evolution in how individual students approach their team-working experiences. Equally, students are perhaps increasingly conscious of, and wary of a possible lack of (emotional) engagement of their fellow team members.

\subsection{Time, personal time and stress}

Time is one of the most strongly emerging themes in 5th year reports from the 2019/2020 cohort. Time issues are mentioned by $73 \%$ of the more recent cohort compared to $58 \%$ previously. As well as time issues being more present with 5th year students, many 3rd year comments also relate to this point. Equally, the nature of time related comments has widened, including; time together with the team;(difficulties relating to) adapting to the school/project timing; too much time (project length), waiting time; wasted time, time to talk and coordinating time; the "rush" culture and mis-managed time. This strongly emerging theme appears to be linked into growing levels of stress among students, with stress mentioned by $47 \%$ of the recent 5 th year cohort, compared to only $12 \%$ seven years ago. Coping with stress issues can be linked to effective time management [11]. More generally, increasing anxiety, feelings of time pressure, stress and mental health issues among students are increasingly prevalent [12], and have been linked to fear of failure [13] as well as a shift from prizing learning as an end in itself to focussing on a job market which may be uncertain and joyless.[12]

Certain aspects of the theme of time can be linked to the above theme of passion, motivation and enjoyment. One student who reported a very positive first experience of team-working, (cohesion and very friendly atmosphere) does not criticise working every day from $6 \mathrm{pm}$ to midnight on the project, and from $5 \mathrm{pm}$ to early morning during the final week of two stages. Frustrations can perhaps be linked to expectations related to enjoyment and time ownership. Students criticise losing personal or free time "...not being able to take advantage of the holidays to go to see our families once every two months because of the workload is not nice." Also, compared to the earlier cohort, very few comments relate to social activity with the group outside of project work.

Differing time investment is a theme that appears to be generating conflict "we had to spend lots of time explaining" to 3rd years, and 3rd years mention problems with 5th years "imposing their timing on the daily schedule". Issues appear to be linked to having control of time. Students generally give emphasis to setting goals and priorities in time management [14], whereas these aspects are only part of time management and do not have marked incidence on final grades. Time planning accompanied by a perception of control over time may better correlate with higher grades. Student reports reflect this, with emphasis on time planning, but indicating feeling not having control over their time. 
Finally, fewer students replied to the recent study despite equal time allocated for replying. Reports were also slightly shorter, suggesting less willingness or less time self-allocated for reflecting on learning. This emerging theme deserves more research to better understand related issues and evolution.

\section{CONCLUSIONS}

This research highlights how the inter-year team-work modules still represent a valuable learning experience, progressively giving students tangible team-working skills. But findings show that certain issues identified in previous research can still be improved. While results suggest that teaching now supports better understanding of certain team-work skills such as communication, planning/organisation, reflection on personal experiences, there is a growing need for support in relation to conflict management, and the emerging issues that may generate this team conflict such as growing heterogeneity in the student cohort, attitudes on time management, personal time and enjoyment.

While communication is generally effective, it may be useful to make students more aware of how the views and experiences within a team can vary considerably. Equally it remains important to help students to appreciate the value of negative team-work experiences [6] for overall learning. While the school has already focused on improving attitudes towards "management", the problem of hierarchies within teams remains an issue that teachers and students need to be vigilant towards and may necessitate renaming the roles within teams. Finally this research highlights the evolving nature of the design student cohort, in relation to perceived time pressure, personal time and their link to positive or negative emotional attitudes, which may need to be better taken into account for effective teamwork. A future challenge will be to keep the value of these teamwork modules, while insuring a more positive overall experience, and ensuring reflection on learning in increasingly time stressed student cohorts.

\section{REFERENCES}

[1] Baard V. and Buchanan J. "Team X" - Developing a Teamwork Experience to Support 'Teamwork Capable' Student Employability, 2018, at: https://teche.mq.edu.au/2018/10/team-x-the-design-and-development-of-a-teamwork-experience-to-support-teamwork-capable-student-employability/

[2] Rauth I., Köppen E., Jobst B. and Meinel C. Design Thinking: An Educational Model towards Creative Confidence, in Proceedings ICDC 2010, Kobe, Japan, 11, 2010.

[3] Tucker R. Teaching Teamwork in Design: A Framework for Understanding Effectiveness in Student Teams, in Tucker R. ed. Collaboration and Student Engagement in Design Education, IGI Global, USA, 2017, pp.1 - 27

[4] Author. Inter-Year Teamwork Formats examined Through Student Experience. Proceedings of E\&PDE 2012, Design Education for Future Wellbeing, Antwerp, Belgium, September.2012

[5] Author. Consecutive Inter-Year Team Projects - A Learning Experience Entity? Proceedings of E\&PDE 2013, Dublin Institute of Technology, Dublin, Ireland, September 2013

[6] Tucker R. and Abbasi N. Bad Attitudes: Why design students dislike teamwork. Journal of Learning Design, Vol.9 No.1, 2016

[7] Kayes D.C. and Kolb D.A. Developing Teams Using the Kolb Team Learning Experience, in Simulation and Gaming, Vol. 36 No. 3, September 2005 pp1-9

[8] Karapanos E., Martens J-B. and Hassenzahl M. On the Retrospective Assessment of Users' Experiences Over Time: Memory or Actuality? Proceedings of CHI 2010, GA, USA, 04,10, 2010

[9] Behfar K.J., ,Peterson R.S., Mannix E.A. and Trochim W.M.K., The Critical Role of Conflict Resolution in Teams: A Close Look at the Links Between Conflict Type, Conflict Management Strategies, and Team Outcomes, Journal of Applied Psychology, Vol. 93, No. 1, 170-188, 2008

[10] Jeno L.M., Raaheim A., Kristensen S.M., Kristensen K.D., Hole T.N., Haugland M.J. and Maeland S. The Relative Effect of Team-Based Learning on Motivation and Learning: A Self Determination Theory Perspective, in CBE-Life Sciences Education, 16:ar59, 1_12, Winter 2017

[11] Lin, Pan and Ching, Common problems and coping solutions of university students in Taiwan, International Journal of Research Studies in Education, January, Volume 4 Number 1, 3-16 2015

[12] Shackle S. The way universities are run is making us ill, at https://www.theguardian.com/society/ 2019/Sept/27/anxiety-mental-breakdowns-depression-uk-students [Accessed February 2020]

[13] McCardle J. Performance Metrics: Are the risks too high to be creative? E\&PDE, UK, 2018

[14] Adams R.A. and Blair E. Impact of Time Management Behaviours on Undergraduate Engineering Students' Performance, SAGE Open, Jan-March 2019: 1-11, 
journals.sagepub.com/home/sgo 